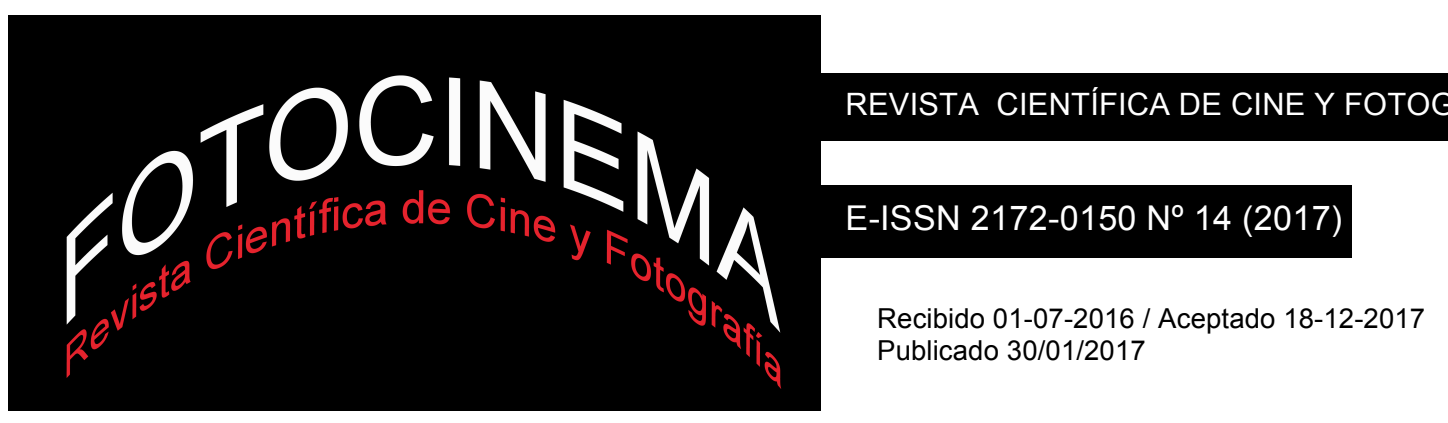

\title{
INFANCIA Y DESESTRUCTURACIÓN FAMILIAR EN EL CINE DE HIROKAZU KOREEDA: NADIE SABE, MILAGRO Y DE TAL PADRE, TAL HIJO
}
CHILDHOOD AND FAMILY DESTRUCTURING IN THE FILMS OF HIROKAZU KOREEDA: NOBODY KNOWS, I WISH \& LIKE FATHER, LIKE SON

\section{Resumen:}

Este artículo aborda el tratamiento de la infancia en las películas de Hirokazu Koreeda Nadie sabe, Milagro y De tal padre, tal hijo. Herederas directas del género del primer drama doméstico japonés (homu dorama), las tres forman un corpus homogéneo al explorar desde el punto de vista infantil el fenómeno de la desestructuración familiar, motivo argumental principal en la filmografía del cineasta japonés. A través de un análisis textual que se apoya en escenas particularmente expresivas, el texto explora primero cómo los niños protagonistas afrontan física y emocionalmente esta vivencia traumática para ellos: el aislamiento frente a sus padres, la añoranza de los mismos y el afán de regenerar los lazos familiares son constantes de estos filmes. En segundo lugar, el artículo analiza dos arquetipos que resultan de la desestructuración familiar: el adulto inmaduro y el niño-adulto. Por último, la mirada cómplice de Koreeda con los niños se advierte en la capacidad ejemplar que les concede en los relatos fílmicos para establecer entre sí fuertes lazos afectivos. Un hecho que refuerza la constante temática en su cine de considerar a la familia como una institución sustentada sobre lazos afectivos, los cuales prevalecen sobre los estrictamente sanguíneos.
Pablo Echart

Universidad de Navarra, España pechart@unav.es

Miguel Muñoz-Garnica Universidad de Navarra, España mmunozg@alumni.unav.es

\section{Abstract:}

This article examines how childhood is adressed in the Hirokazu Koreeda's films Nobody Knows, I Wish and Like Father, Like Son. Being identifiable as inheritances of the original Japanese homu dorama -or home drama- films, they constitute an homogeneous corpus to explore the issue of family destructuring from a childish perspective. This issue happens to be a main plot motif in the filmography of the japanese director. Using a textual analysis supported by particularly expressive scenes this article explores, first, how the protagonist children physically and emotionally face this traumatic experience: their isolation before their parents, their yearning for them and their efforts to regenerate the family ties. All of these are constant tropes in the identified films. Second, this article analyses two archetypes produced by family destructuring: the inmature adult and the adult-child. Finally, Koreeda's knowing look to children is made clear in the exceptional capacity of creating strong bonds that he attributes to them. This fact reinforces another thematic constant in his ouvre: the conception of family as an institution supported by affective more than blood ties.

Palabras clave: Koreeda; drama doméstico - homu dorama; infancia en el cine; desestructuración familiar; cine japonés.

Keywords: Koreeda; Home Drama - homu dorama; Childhood on Film; Family Destructuring; Japanese Film. 


\section{Introducción}

Hirokazu Koreeda ha sido identificado como el cineasta de su generación que ha mostrado una preocupación más profunda y continua por representar la sociedad japonesa contemporánea (Joo \& Park, 2013), tarea que sigue llevando a cabo desde la predilección por las temáticas y conflictos familiares. Esta recurrencia hace natural su adscripción al drama doméstico o homu dorama, considerado por Anderson y Richie como un subgénero del shoshimingeki, esto es, el género surgido en los años treinta del siglo XX (y en auge hasta principios de los sesenta) alrededor de personajes de las clases trabajadoras japonesas y sus historias cotidianas. Si bien el homu dorama se ha desarrollado posteriormente como género televisivo, estos dos autores sitúan su origen en el ámbito cinematográfico:

El shoshimingeki y sus géneros descendientes, las películas de madres (hahamono) y las películas de esposas (tsuma-mono) han confluido en el drama doméstico (homu dorama). Se trata del género que ha eclipsado a todas las demás posibilidades de contar historias sobre la vida contemporánea. El trabajo colectivo de los viejos maestros de la Shochiku como Shimazu, Gosho, Ozu y Naruse representa las glorias del primer drama doméstico ${ }^{1}$. (Anderson y Richie, 1982, p. 451).

La herencia que se sugiere en este artículo, por tanto, es la de Koreeda como renovador de ese primitivo homu dorama cinematográfico, un género que, como apunta Sato (1987, pp. 139-144), sufrió una deriva hacia el escapismo y la dulcificación de la realidad social en su posterior desarrollo televisivo que lo distanció del realismo del shoshimingeki. Pues bien, Koreeda es considerado un cultivador de la versión contemporánea del shoshimingeki (Richie, 2005, p. 239) y heredero de maestros del género como Yasujiro Ozu (Desser, 2007; Caro-Oca \& López Rodríguez, 2011) o Mikio Naruse, este último señalado por el propio cineasta como una influencia en parte de su filmografía (Schilling, 2011, p. 12).

Las tres películas escogidas se adscriben al drama doméstico: Nadie sabe (Dare mo shiranai, 2004), Milagro (Kiseki, 2011) y De tal padre, tal hijo

\footnotetext{
${ }^{1}$ Las traducciones del inglés corresponden a los autores.
} 
(Soshite chichi ni Naru, 2013). Centradas en el universo infantil, las tres presentan una fuerte cohesión narrativa y temática que se convierte en el objeto de análisis de este artículo. Como se verá en el primer epígrafe, este carácter unitario obedece en buena medida a que todas ellas exploran las consecuencias emocionales -y en la primera, también las materiales y físicas (Jacoby, 2011, pp. 66-83)- que experimentan los niños al fracturarse el núcleo familiar en el que han vivido. En el segundo epígrafe se estudia cómo los conflictos infantiles vienen provocados por la falta de responsabilidad que demuestran unas figuras paternas inmaduras, lo cual obliga a los niños a asumir unos roles impropios de su edad: exploramos así los rasgos distintivos de lo que hemos denominado como el "niño-adulto". Por último, un poderoso rasgo de confluencia de la imagen infantil en estos filmes consiste en la afinidad natural que se da entre los niños, lo cual contrasta con la inestabilidad de las relaciones adultas y sirve a un propósito temático recurrente en la filmografía de Koreeda: la creencia en la formación y regeneración de la familia a partir de lazos afectivos surgidos en la convivencia, estimados como más fuertes y genuinos que los establecidos por la consanguinidad.

Para llevar a cabo esta investigación se emplea como metodología un análisis textual focalizado en la construcción dramática de los personajes protagonistas y de las relaciones que estos establecen con los personajes que los circundan. Este enfoque se refuerza con el análisis de la composición visual de ciertos planos y escenas que ilustran particularmente bien los asuntos abordados. Algunas de estas imágenes han sido recogidas en los fotogramas seleccionados.

\section{La desestructuración familiar como eje narrativo}

La ausencia representa un motivo argumental esencial de la filmografía de Koreeda (López-Rodríguez, 2013, p. 7; McDonald, 2006, pp. 198-218; Fecé, 2003, pp. 175-195). Los tres filmes analizados concretan esta constante en torno a alguna forma de desestructuración familiar. Coinciden así con otros 
dramas familiares del cineasta que, como Maborosi (Maboroshi no Hikari, 1995) o Still Walking (Aruitemo, aruitemo, 2008), examinan desde un punto de vista adulto las heridas afectivas derivadas de la pérdida de un ser querido -un esposo en el primer caso, el primogénito de la familia en el segundo- en los miembros de su familia y en la forma en que éstos se relacionan entre sí. Los últimos dramas familiares de Koreeda estrenados en salas hasta la fecha no son una excepción. Nuestra hermana pequeña (Umimachi Diary, 2015) otorga su protagonismo a cuatro jóvenes hermanastras privadas durante su crecimiento de la tutela de un padre o una madre, y marcadas por tanto como afirma la mayor de ellas- por una infancia robada. Por su parte, Después de la tormenta (Umi yori mo mada fukaku, 2016) narra la relación de un hombre divorciado de mediana edad con su exmujer y su hijo, pero también con su madre y la memoria de su padre, creando un discurso muy rico sobre las relaciones intergeneracionales dentro de la familia y los errores cometidos por los padres. A propósito de Nuestra hermana pequeña, resulta ilustrativo cómo el propio Koreeda reconoce su afinidad personal hacia unas historias en las que la fractura familiar deja espacio, en ocasiones, a ciertas vías de regeneración, tal y como se verá después: “Me parece importante contar una historia sobre una familia en la que algunos de sus miembros ya no están. Pero en las que hay alguien más tratando de tomar el relevo del rol de los padres, tratando de reconstruir esos lazos familiares. Me encantan ese tipo de historias. Me conmueven mucho” (Bradshaw, 2015).

En Nadie sabe, Milagro y De tal padre, tal hijo, la fractura familiar no resulta de la irrupción de la muerte sino de una decisión voluntaria tomada por los padres o madres. Interesado en primer término en la exploración de los conflictos infantiles, Koreeda incide en explorar la distancia que separa a padres e hijos como consecuencia de que los primeros prioricen sus intereses sobre los de los segundos, y se muestren poco sensibles a sus afectos y necesidades. En Nadie sabe, cuatro hermanastros son abandonados a su suerte por su madre común, que se establece en otra ciudad por motivos sentimentales y se limita a enviarles algo de dinero esporádicamente; en Milagro, un niño pre-adolescente sufre por no poder convivir con su padre y 
su hermano, y su mayor aspiración es la de reunificar su familia, dividida tras la separación matrimonial de sus padres; por último, De tal padre, tal hijo ilustra la dureza del desarraigo familiar en dos niños de seis años que son paulatinamente separados de los padres con los que han crecido desde su nacimiento para, en cambio, ser devueltos a sus respectivos padres biológicos. Procedemos a mostrar cómo este eje narrativo común de la desestructuración familiar opera en cada una de las tres películas.

\subsection{Nadie sabe}

Basado en unos conocidos hechos reales de 1988, Nadie sabe es el film de tono más lúgubre, el que pone mayor énfasis en el egoísmo irresponsable de los padres y en su falta de empatía con sus hijos. El relato se estructura sobre el progresivo deterioro de las condiciones en las que viven cuatro hermanastros en un apartamento en el distrito de Sugamo, en Tokio, una vez que su madre los abandona y examina cómo afronta esta ausencia cada uno de ellos. El punto de vista principal es el del mayor, Akira, un niño de doce años que asume la difícil tarea de preservar la unión de los cuatro. Responsable y atento, Akira se enfrenta a dos grandes obstáculos: uno de carácter material -la falta de recursos económicos- y otro de carácter afectivo -el desengaño que experimenta en relación con su madre-.

El primero de ambos se refleja en la depauperación de la calidad de vida de los hermanastros. Al hecho inicial de verse obligados a permanecer confinados en el apartamento para no llamar la atención se suma una alimentación precaria; la pérdida de bienes básicos como son el agua, el gas o la luz; y un consiguiente deterioro higiénico. Sobre el aspecto afectivo, es significativo que, de entrada, Akira interioriza la fantasía de su madre de formar una familia junto a su nueva pareja, de manera que los seis pudieran convivir en armonía y con normalidad (es decir, por ejemplo, que los cuatro niños pudieran ir al colegio). Después, al experimentar como una dolorosa revelación la falsedad de esta promesa materna y al hacerse de esta manera evidente a sus ojos la imposibilidad de formar una nueva familia, Akira claudica de sus responsabilidades adquiridas: es el tiempo de evadirse en el refugio del juego y en la compañía de unos amigos dudosos, y el único 
momento en el que surgen desavenencias con sus hermanastros. La ruptura definitiva del vínculo materno-filial, que acontece en la estremecedora escena de una cabina telefónica que consume las últimas monedas de Akira, le aproxima al robo. Los efectos perniciosos de la ausencia paternal se reconocen también en su amiga Saki, otra chica solitaria y privada de afectos que llega a coquetear con la prostitución para conseguir dinero.

La gran aspiración de Akira, como la del protagonista de Milagro, es formar parte de una familia completa, estructurada en torno a la unión sólida entre padres e hijos. Si bien se satisfaría con que el novio de su madre ejerciera el rol paterno, Akira aspira de manera velada a reencontrarse con su padre biológico. Koreeda expresa esta idea a través de la recurrencia visual a unos aviones a los que concede un valor metonímico, y al motivo visual de unas manos adultas que, por un instante, arropan las del niño -imagen que sintetiza su necesidad de ser atendido y cuidado ${ }^{2}-$ en un momento culminante del relato.

El hecho de que las únicas atenciones que reciben los niños provengan de personajes más bien anónimos subraya el fracaso de las figuras paternas en el ejercicio de sus obligaciones elementales. La madre es presentada como un personaje infantil y egoísta. Y los hombres tampoco son presentados de una forma positiva: el padre de Akira abandonó a su mujer y es una figura ausente; y los dos posibles padres de Yuki -la más pequeña de los cuatroeluden cualquier compromiso entregándole a Akira una suma miserable de dinero.

Según mencionábamos, Nadie sabe también atiende al modo en que cada uno de los otros tres pequeños experimenta la ausencia materna. Antes que en la palabra, Koreeda se apoya en las miradas expectantes y dolidas que los dos hermanos mayores dedican a su madre, y en el vínculo de cada hermano a ciertos objetos. Así, Kyoko, la segunda en edad, impide a Akira que venda las ropas de su madre, incapaz de aceptar el hecho de que ella no volverá;

${ }^{2}$ No es la única imagen que opera en este sentido: lo mismo cabe decir, por ejemplo, de la escena temprana en la que Akira simula estar dormido para ganarse así la atención de su madre. 
además, un piano de miniatura concentra su deseo de alcanzar una apacible cotidianeidad doméstica (el objeto funciona como sinécdoque de su padre ausente, ya que el único dato que conoce de él es que era músico), y su manicura expresa la nostalgia por una madre que se desvanece como el color de las uñas (más adelante nos detendremos en la importancia de este motivo visual). Shigeru, de unos ocho años, es una especie de "pequeño salvaje": un niño que, sin restricciones parentales, se refugia en las satisfacciones primarias de la comida y los juegos. Yuki, la más inocente, deposita con su mirada implorante la confianza en Akira. Al ser la más pequeña es también la más frágil, y su suerte en el relato constituye la mayor apelación a la injusticia cometida sobre estos niños por parte de sus padres.

De la mano del personaje de Saki, la chica solitaria -otra encarnación de la ausencia paterna, esta vez en un estrato social superior- que se termina yendo a vivir con ellos, Nadie sabe introduce otro elemento recurrente en la filmografía de Koreeda, y que será objeto de análisis en el tercer epígrafe: la capacidad de mutación y regeneración familiar a partir de la inclusión de nuevos miembros que son aceptados como tales gracias a los afectos que genera la convivencia. Quizá la afirmación más bella de esta idea se encuentra en Still Walking, cuando una mujer casada en segundas nupcias le dice a su hijo que la mitad de su naturaleza es de su padre biológico y la otra mitad de ella, y al preguntar entonces el niño por el papel de padrastro, ella responde que éste también se irá colando en él poco a poco pero con firmeza.

\subsection{Milagro}

Frente a la dureza de la desestructuración familiar en Nadie sabe, Koreeda adopta un tono más amable en Milagro, que no en vano se constituye como relato de formación agridulce. Como en Nadie sabe, el film se estructura alrededor del conflicto surgido de la imposibilidad de que se materialice el anhelo de un niño preadolescente -Koichi, también de doce años- de recuperar la unidad familiar, en este caso truncada por la reciente separación matrimonial de sus padres; como consecuencia, Koichi vive con su madre y abuelos, mientras que su hermano pequeño, Ryu, lo hace con su padre. 
Koreeda plantea diversas estrategias narrativas para subrayar el carácter obsesivo de este anhelo. Muy notoriamente, la trama externa del relato se concibe sobre este asunto: Koichi se reúne con Ryu en el punto exacto de la geografía japonesa en el que dos trenes-bala se cruzan, pues según se rumorea, la energía que ahí se provoca es capaz de producir el milagro que cada cual formule, y que evidentemente en su caso es la reconstrucción de su familia. Para conseguirlo, Koichi supera distintos inconvenientes, incluidos el logro del dinero para los billetes de tren y una charada para escapar del colegio. No menos gráfico, Koichi reza al volcán que preside Kagoshima, donde ahora vive, para que desate toda su violencia, obligue a evacuar la población y haga posible así el reencuentro de los cuatro. Además, pese a su credulidad, Koichi es, como Akira, otro niño responsable y maduro. Con determinación alecciona al pequeño Ryu para que vigile que su padre no inicie una relación sentimental, al tiempo que procura en vano engañarle a éste sobre un posible romance de la madre con otro hombre. También sueña -literalmente- con la reunificación de los cuatro y proyecta su conflicto cuando contempla felices escenas familiares ajenas. Tampoco es baladí que prefiera olvidar las discusiones que tenían sus padres, a diferencia de Ryu, para quien resultaban insoportables.

En su viaje al encuentro de los trenes-bala, Koichi, Ryu y los amigos de ambos son acogidos durante una noche por un matrimonio de ancianos, en lo que constituye una secuencia de alto valor simbólico y semántico. Al menos tres aspectos son aquí destacables: la nostalgia de una domesticidad feliz, la bendición que representan los niños para los ancianos, y la sintonía que se establece entre unos y otros. Efectivamente, llama la atención la facilidad con la que, en escasas horas de tiempo fílmico, se forma una sensación armoniosa de familia entre los ancianos y los niños, de manera que la casa resplandece como un espacio de convivencia apacible y gratificante para todos ellos. Como antítesis de los padres que ven a los niños como cargas para ejercer su libertad (Nadie sabe), los ancianos se sienten agraciados por esta visita inesperada de unos muchachos que hacen contagiosas su alegría e ilusión, y que además les permite restañar una vieja herida provocada por la enésima 
falta de entendimiento entre unos padres -ellos mismos-y una hija que hace tiempo los abandonó. En definitiva, se establece entre los ancianos y los niños una sintonía que está también presente en la relación cómplice de Koichi con su abuelo, otro personaje marcado por un fuerte deseo. Este anciano y el matrimonio que acoge a los niños se presentan como personajes próximos a sus anhelos, frente a unos padres ausentes o que siguieron su propio camino y unas madres que, siendo amables con sus hijos, no perciben sus deseos más profundos. Por eso tampoco es extraño que la abuela de Koichi presente un perfil desenfadado y algo lúdico (su afición por el baile), que tendrá prolongación en la caracterización de la abuela de Keita en De tal padre, tal hijo. Más clarividentes que los hombres y mujeres de mediana edad, y más cercanos a las coordenadas de la infancia, los ancianos -y en especial las ancianas- se presentan en estas dos películas como depositarios de una sabiduría sobre lo que es verdaderamente valioso en la vida, y que no es otra cosa que el cuidado de los afectos paterno-filiales.

\subsection{De tal padre, tal hijo}

La ruptura de la unidad familiar, el conflicto que este acontecimiento provoca en los niños y la distancia entre padres e hijos vuelven a ser constantes en De tal padre, tal hijo, de manera que existe una fuerte vinculación de este film con Nadie sabe y Milagro. Frente a ellos, en cambio, los matrimonios se presentan como vínculos sólidos y unidos, y la inmadurez (Milagro) o el egoísmo (Nadie sabe) de los padres no actúan como detonantes de la fractura familiar. En esta ocasión obedece a un conflicto establecido entre la filiación de sangre y la filiación afectiva: seis años después de los nacimientos de sus hijos, dos familias conocen que éstos fueron intercambiados en el hospital donde nacieron; con esta revelación surge el dilema: seguir cuidando del niño que han criado o recuperar al hijo biológico mediante un intercambio.

Al decantarse los matrimonios por la segunda opción, el film examina la violencia que esta decisión ejerce sobre los niños que la sufren, Keita y Ryusei. La experiencia del intercambio resulta traumática para ambos, si bien el contraste de caracteres y de valores de los padres varones - uno severo 
y otro infantil y entregado- es importante para que el relato sancione como favorables ciertas formas de educar a un niño y condene otras.

Ryota, el padre que impulsa el intercambio, encarna el éxito profesional y se ha empeñado en educar a Keita a su imagen y semejanza, ignorando las particularidades del niño. Además, su decantación por su hijo biológico (Ryusei) es visto por Keita como una falta de amor hacia él. En contrapartida, este dolor del niño es mitigado por el amor y el afecto que recibe de Yudai y su esposa, Yukari, que lo acogen como a uno más de los tres hijos que tienen. Aunque glotón y algo desastrado, Keita estima en Yudai su cercanía y cariño, que devuelve con palabras de aprecio y gestos de gratitud. Koreeda se apoya en el motivo verbal de las cometas para recordar que es el tiempo de juego compartido con los niños lo que genera vínculos genuinos entre padres e hijos.

Pese a las comodidades materiales de que dispone, y pese a la atención y cariño que le dispensa la madre de Keita -el papel de las dos madres es equivalente, y de hecho el relato traza una corriente de sintonía entre ambas-, Ryusei no se adapta a su nuevo hogar. La orden de Ryota a su hijo biológico para que les llame "papá" y "mamá" a él y a su esposa es desacatada por el pequeño, poniendo de manifiesto la tesis fundamental del relato: que los vínculos paterno-filiales no pueden imponerse, sino que éstos solamente pueden surgir del cultivo de los afectos. Esta postura es defendida por la madre de Ryota, que considera que la identificación paterno-filial surge de un proceso similar al que experimenta un matrimonio, y que por tanto no obedece a razones de sangre sino que surge de la convivencia: "Quieres a la otra persona y acabas siendo como ella"3. Ayudado por la presencia obstinada de Ryusei, Ryota flexibiliza su rigidez, se aproxima al niño a través del juego, y acaba abrazando la visión del parentesco que le da su madre y que sanciona favorablemente el relato. Gracias a ella "recupera la cordura" para poder recuperar a Keita. De las tres películas analizadas, De tal padre,

3 De las tres películas aquí tratadas, Milagro es la que hace más evidente esta idea: Koichi asume la personalidad sufridora y atormentada de su madre, mientras que el pequeño Ryu se reconoce en la despreocupación y egocentrismo de su padre. 
tal hijo es el caso más evidente en el que queda salvada la desestructuración familiar que sus guiones comparten como punto de partida.

\section{Las relaciones entre mundo infantil y mundo adulto}

Recapitulando, las tres películas estudiadas comparten una desestructuración familiar ocasionada por una actitud egoísta de los padres, que anteponen sus intereses sentimentales o laborales a los de sus hijos. Esta actitud queda especialmente patente en los rasgos de inmadurez que se esbozan en la caracterización de esos personajes adultos. A su vez, este atributo provoca que los niños tengan que asumir ciertas funciones que, por edad, no les corresponderían y les empuja a una madurez forzada. Con lo que tiene lugar una suerte de hibridación intergeneracional de roles que es muy característica del cine de Koreeda: las figuras del adulto inmaduro y el niñoadulto. Procedemos a profundizar en ambos aspectos.

\subsection{La inmadurez de los padres}

El personaje más reconocible en esta actitud es Keiko, la madre de los cuatro hermanos de Nadie sabe. Su inmadurez se manifiesta principalmente en su negligencia en el cuidado de sus hijos, como evidencian sus ausencias cada vez más prolongadas del piso familiar o el llegar bebida a altas horas de la noche. Resulta perceptible en la primera parte del film una inversión de roles que vincula a Keiko con la despreocupación y la diversión propia de una adolescente, y a Kyoko y sobre todo Akira, los dos hijos mayores, con las responsabilidades adultas y paternales. Es muy ilustrativo, por ejemplo, que Akira y Kyoko manifiesten en sendas ocasiones su deseo de volver al colegio y que sea Keiko la que les disuada de hacerlo.

La escena que Keiko protagoniza con Akira en el balcón ejemplifica bien esta inversión de roles. A modo de confesión, con un lenguaje corporal (baja la mirada y encoge la postura) y una entonación que remite a un candor preadolescente, ella le confiesa que está enamorada (F1). La respuesta de Akira, enunciada con un tono desganado, es muy expresiva: “¿Otra vez?”. Se hace patente de esta forma el contraste entre este escepticismo inicial de 
Akira (más propio de un adulto) y la ilusión fantasiosa de su madre (más propia de una adolescente sin experiencia vital). En este mismo sentido opera otra escena con los dos personajes: Keiko se prepara para salir de noche. Apoyado en el marco de una puerta imitando los modos de un padre que pretende controlar a su hija, Akira le pregunta a qué hora regresará esta vez; Keiko, mientras se maquilla, responde con una evasiva y a continuación pregunta qué va a haber para cenar.

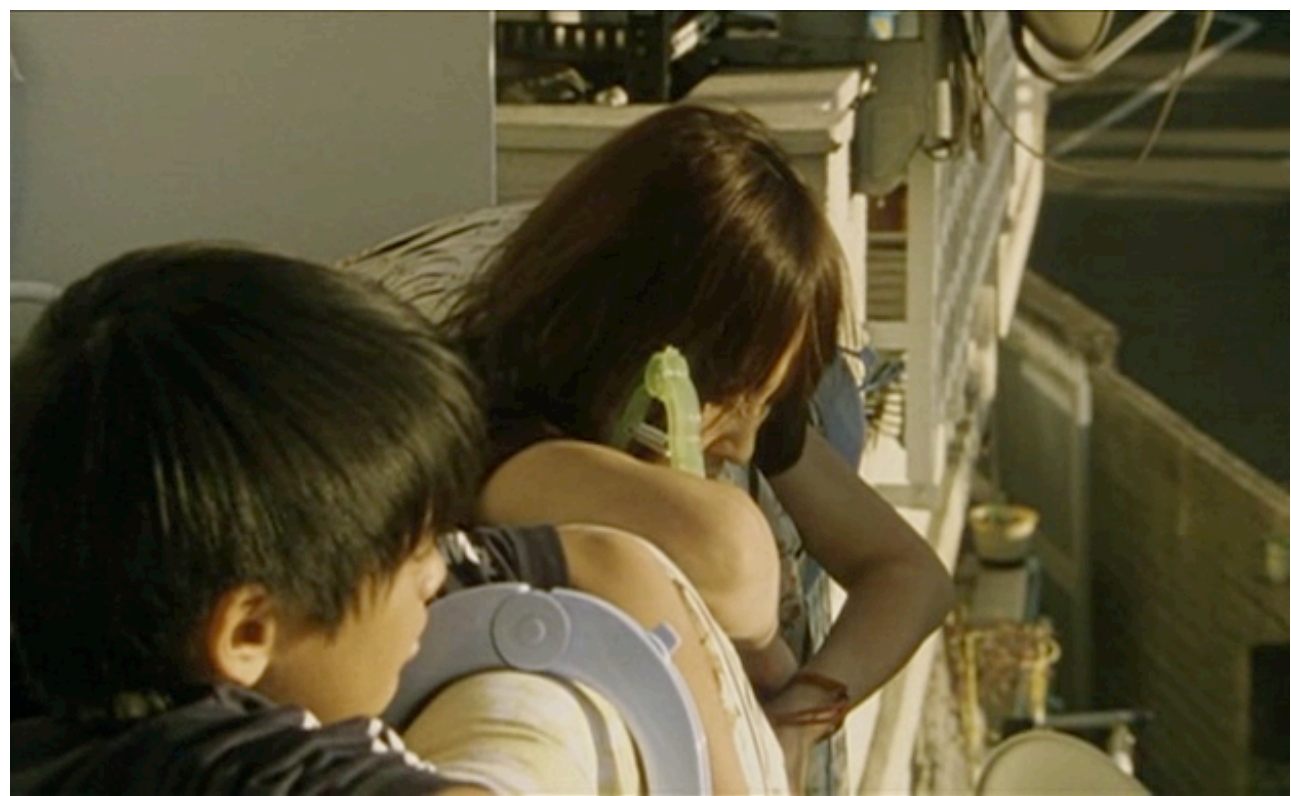

F1. Nadie sabe (Dare mo shiranai, 2004)

Existe una inversión de roles igual de patente, si bien filtrada como comedia, en la relación entre Ryu, el hermano menor coprotagonista de Milagro, y su padre. Ya en la presentación de los dos se hace evidente que Ryu ha desarrollado una amplia autonomía y que es él quien asume las tareas domésticas. Incluso se encarga de despertar a su padre y recordarle que tiene que ir a trabajar, ante la actitud perezosa de este. Esta inversión queda especialmente explícita en una escena en la que el padre vuelve a casa de madrugada y Ryu le espera en el salón con una postura y una mirada de severidad que remiten a lo paternal, así como el hecho de que le regañe por su irresponsabilidad mientras las palabras y el lenguaje corporal del padre señalan que acata la autoridad del hijo. Este sometimiento queda subrayado 
por la forma de presentar el plano, con las figuras de perfil para recalcar sus posturas y las alturas a las que se sitúan sus miradas (F2).

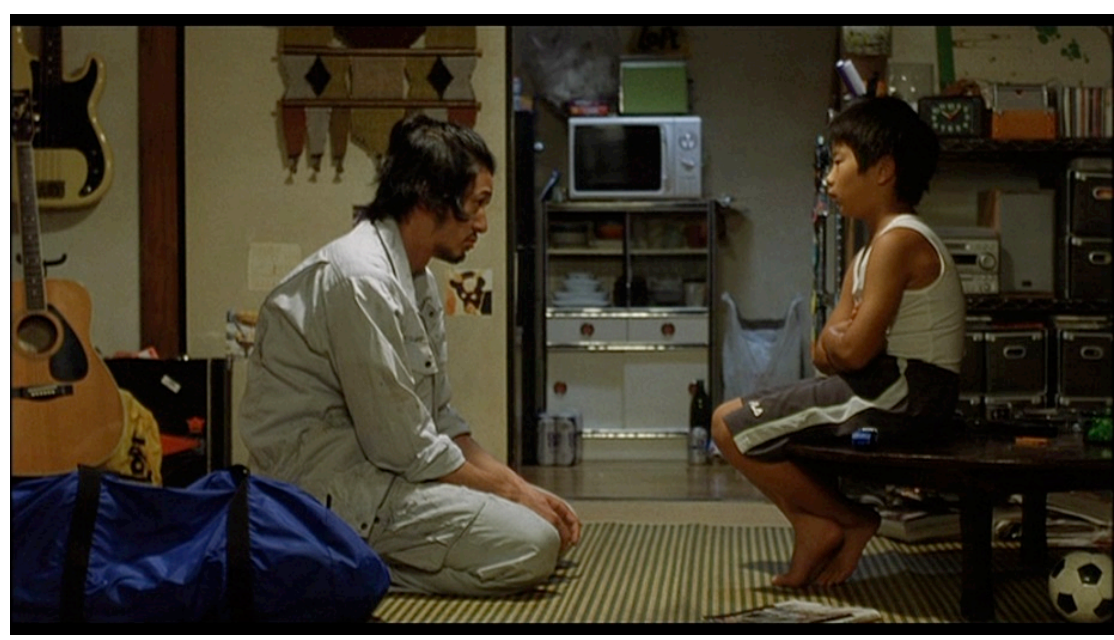

F2. Milagro (Kiseki, 2011)

En De tal padre, tal hijo no está presente esta inversión de roles, pero sí se explora la inmadurez de un personaje adulto: Ryota, el padre de clase mediaalta. En este caso, no se trata de una regresión hacia comportamientos infantiles o adolescentes, sino de la falta de una madurez afectiva. Como hemos mencionado, el conflicto planteado en la película enfrenta la importancia de los lazos sanguíneos frente a las vivencias compartidas como fuente de los vínculos afectivos. Hay una clara toma de partido por la segunda opción en el discurso de fondo que plantea Koreeda, a la que se opone la postura de Ryota, que prioriza lo sanguíneo al decidir a cuál de los dos hijos criar. La película narra su conciliación con la postura afectiva mediante una trama de maduración sentimental.

El hecho de que sea el personaje adulto el que experimenta esta trama de maduración plantea una inversión respecto a la tendencia narrativa dominante de emplazar este tipo de tramas en el territorio de la infancia o de la adolescencia. Hay, por supuesto, más ejemplos de tramas de maduración adulta. Pero su apariencia, a priori, de película centrada en la infancia hace llamativo este detalle. La propia Milagro sigue este criterio habitual, tan común en la misma cinematografía nipona: el niño abandona su zona de confort egocéntrico de la infancia para descubrir una faceta del mundo 
desconocida (la presencia del "otro" y los modos de relacionarse con él) y adaptarse a ella. Es más, cuando Ryota decide llevar a cabo el intercambio de niños le plantea a Keita (su hijo no biológico) el cambio a la nueva familia como una misión en la que debe demostrar lo fuerte que es para cumplirla con éxito. Es decir, considera la experiencia un catalizador de crecimiento personal para Keita. Sin embargo, según exponemos, es el propio Ryota quien termina protagonizando ese crecimiento al verse obligado a salir de su zona de confort (la adicción al trabajo) para abrirse a un mundo de afectos familiares que hasta entonces prácticamente desconocía. Es él mismo el que termina rogando a Keita que le perdone su error tras haberlo asumido.

Koreeda visualiza esta idea en la penúltima escena de la película, de clara lectura metafórica. En un parque, Ryota y Keita caminan a la par, aunque cada uno de ellos en un camino paralelo. Esta decantación escenográfica, que ilustra visualmente la separación que se ha instalado entre ellos, se refuerza con el empleo de una serie de planos contraplanos, recurso fotográfico que en efecto los confronta. Mientras caminan, Ryota expone sus disculpas a su hijo. La escena termina en un plano general que recoge el punto de convergencia de los dos caminos, donde sucede la reconciliación. Alcanzada esta, Ryota adopta una posición en el plano que dialoga en cierto modo con la escena que señalábamos antes de Milagro: se agacha, situándose a la altura física de su hijo por primera vez, mostrando su actitud ahora comprensiva con él y con un mundo infantil que hasta entonces le ha resultado ajeno (F3).

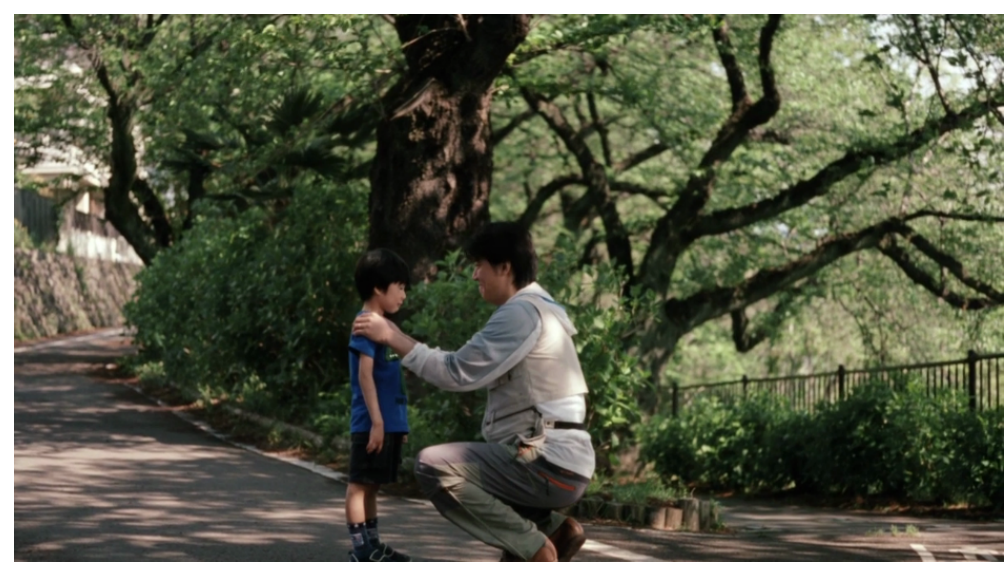

F3. De tal padre, tal hijo (Soshite chichi ni Naru, 2013) 
Hay una diferencia fundamental en esto último con respecto a los casos de la madre de Nadie sabe y el padre de Milagro: mientras que en aquellos la regresión hacia el infantilismo es percibida como algo negativo, en tanto que expresa la inmadurez e irresponsabilidad de estas figuras paternas, en Ryota es justamente su falta de conexión con el mundo infantil la fuente de su inmadurez afectiva. Esta diferencia se hace aún más patente a través de la caracterización de Yudai, el padre de clase media-baja de la familia con la que se realiza el intercambio. $\mathrm{Su}$ principal atributo es precisamente el infantilismo, pero esta vez toma un cariz positivo al plasmarse más como una cercanía comprensiva al mundo de los niños que como un síntoma de inmadurez. Su sintonía con lo infantil se utiliza de forma recurrente como elemento narrativo y visual de contraposición ante la frialdad desapegada y la tendencia autoritaria de Ryota. Este último, a diferencia de Yudai, concibe una educación en la que prima la posición social futura de su hijo sobre la mera vivencia despreocupada de la infancia. Tómese como ejemplo la recogida en la imagen $\mathrm{F}_{4}$ : Ryota, sentado en la mesa donde los adultos dialogan sobre la decisión acerca del intercambio de niños, se niega a acercarse al castillo hinchable donde estos juegan. La siguiente toma, en contraplano, muestra cómo Yudai asalta el castillo y juega con ellos, creando un opuesto claro con la lejanía de Ryota. Es además, un tipo de escena que se repite en varias ocasiones.
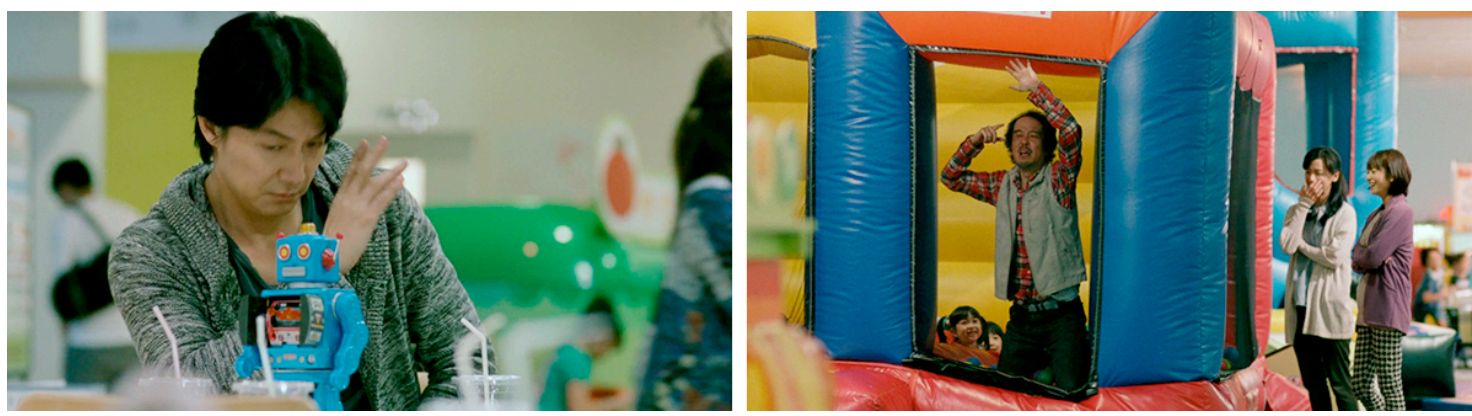

F4. De tal padre, tal hijo (Soshite chichi ni Naru, 2013) 


\section{2. La figura del niño-adulto}

La inmadurez de los padres y el intercambio de roles con los hijos dan lugar a otra figura muy llamativa en el cine de Koreeda, a la que proponemos denominar el niño-adulto. Esto es, el niño que asume ciertas responsabilidades y actitudes que por edad no le corresponden, pero a las que se ve forzado por las carencias derivadas de la negligencia de sus padres.

El caso más claro es Akira, el hermano mayor protagonista de Nadie sabe. El intercambio de roles que tiene con su madre resulta especialmente perceptible tras el abandono de la misma del hogar familiar. Desde ese momento, el hermano mayor se arroga con tareas propias de un cabeza de familia adulto: lleva las cuentas económicas de la casa, sale a hacer la compra, dirige la convivencia... Y, sobre todo, se erige como el protector del bienestar emocional de sus hermanos. En una escena a mitad de la película, cuando ya han pasado meses desde el abandono de la madre, compra regalos de Navidad para todos sus hermanos, los envuelve, pide a una dependienta que les escriba dedicatorias haciéndose pasar por su madre y los lleva a la casa. Justifica además la ausencia de ésta (que había prometido que pasaría la Navidad con ellos) por motivos de trabajo, pese a que en la última secuencia en la que ha visto a su madre ya había dejado clara su desconfianza hacia sus excusas. Por tanto, son actos que no obedecen a un deseo de excusar a su madre, sino de atender a las necesidades afectivas de sus hermanos haciéndoles sentir que siguen siendo queridos por ella.

Por su parte Kyoko, la hermana mayor, desarrolla conductas que evocan los modos maternos: pone las lavadoras, lee cuentos a su hermana menor, etc. Gráfica es la escena en la que, tras la comida y cuando los dos hermanos menores se han retirado de la mesa, Kyoko comenta con Akira que uno de ellos no se ha comido la zanahoria, reproduciendo la manera en la que dos padres hablarían de sus hijos. Pero a la vez Kyoko muestra, de forma mucho más apreciable que Akira, que necesita la presencia de la madre. En la última vuelta casa de ésta tras meses de ausencia, Kyoko le reprocha su desaparición, pero la perdona rápidamente y muestra una menor desconfianza en sus promesas que Akira. El acercamiento recuperado entre 
las dos en este punto de la trama se cuenta en una escena en la que la madre le pinta las uñas a Kyoko, en lo que será lo último que hagan juntas antes de que la primera se marche para no volver. Para recalcar la importancia que tiene este detalle para la pequeña, Koreeda convierte al esmalte de uñas en un motivo visual recurrente. Primero filma el momento compartido de la madre pintándole las uñas, luego a Kyoko ante el tocador de su madre observándose en el espejo mientras toma el bote de esmalte. En ese momento, el bote se le cae al suelo y deja una mancha roja. Transcurridos meses de tiempo fílmico, se vuelve a insertar un plano en el que Kyoko toca el rastro que ha dejado la mancha en el suelo (F5). Con ello, se connotan los sentimientos de vacío que provocan el deseo de imitación de su madre, su recuerdo y la marca de su ausencia.
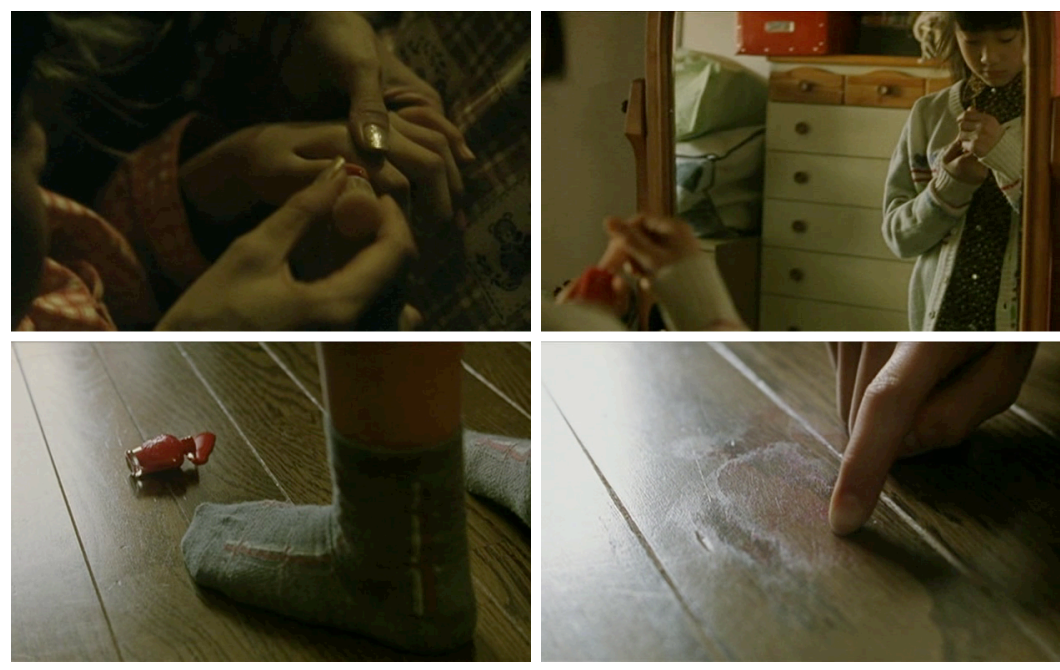

F5. Nadie sabe (Dare mo shiranai, 2004)

Esta doble dimensión de Kyoko (su ejercicio de roles maternales junto a su anhelo de una figura que ejerza esos roles sobre ella misma) está también presente en Akira, y da cuenta de un matiz fundamental en esta figura del niño-adulto: pese a sus rasgos de madurez, no deja de ser un niño que tiene comportamientos y necesidades propias de su edad. Esta dualidad resulta fundamental en Akira porque es el único de los personajes que tiene acceso a los dos universos que configuran la construcción de escenarios de Nadie sabe. Por un lado existe el espacio privado de los hermanos, que está estrictamente acotado al interior del piso hasta casi el final de la película. Y 
por otro lado existe un espacio público al que sólo Akira puede salir, si bien sus acercamientos al mismo son limitados: por mucho que establezca ciertos contactos, no se somete a la normatividad social (ir a la escuela, quedar con sus amigos, desarrollar sus aficiones fuera de casa...) que le haría formar parte integrada de él. De este modo, Akira ejerce un rol patriarcal en el escenario doméstico, mientras que es en el mundo exterior donde se advierte la vulnerabilidad y los anhelos propios de su condición infantil. Significativamente, una de las mayores manifestaciones de su dualidad ocurre cuando entra en contacto directo con el mundo de los adultos más frío y desencantado. Esto sucede en las visitas que hace a los dos posibles padres de su hermana menor en sus lugares de trabajo. Pues bien, el contacto de Akira con la mezquindad de estos personajes viene puntuado por dos comportamientos infantiles: de entrada, mantiene un rápido juego de muecas con un niño desconocido; y, en el plano de cierre de la secuencia, Akira juega solo a baloncesto con una papelera. Así, inconscientemente, Akira se repliega sobre un juego elemental para protegerse de la "cruda realidad" con la que está lidiando.

Este último detalle, además, es lo que hace especial el caso de Nadie sabe en la forma que tiene este título de "eliminar" en cierto modo el mundo adulto. Aunque Akira establece con éste contactos puntuales, Koreeda tiende a tratarlo en fuera de campo, ajeno al punto de vista infantil que la narración adopta, y que desde el mismo es observado con desconocimiento. Por ejemplo, las relaciones de la madre fuera del escenario doméstico están fotografiadas siempre desde el interior del mismo: Akira observa desde la ventana cómo un coche con un conductor inidentificable la deja en la puerta de su casa (F6), o se la ve hablando por teléfono con un interlocutor cuya voz no se escucha. Así, al suspender casi por completo el universo adulto, lo que queda es un universo infantil tendente al encierro en sí mismo y que imita algunos procedimientos del primero adaptados a sus propias necesidades. Ello se traduce esencialmente a la estructura familiar que los cuatro hermanos crean a falta de una familia convencional que les preceda. Donde los roles paternales necesarios para un cierto mantenimiento del orden 
afectivo se combinan con comportamientos infantiles negligentes con respecto, por ejemplo, al orden material de la casa. Esto último no deja de ser una manifestación de que, pese a esta imitación de roles llevada a cabo por los niños, la obligación de actuar como adultos les supera.
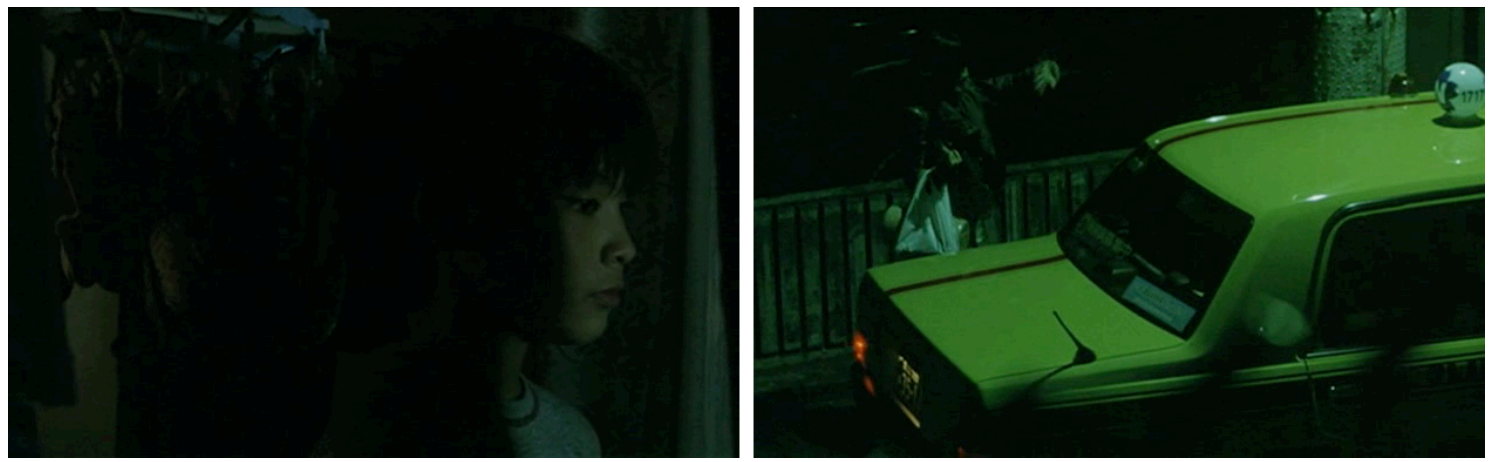

F6. Nadie sabe (Dare mo shiranai, 2004)

La figura del "niño adulto" se aplica en Milagro a los dos hermanos protagonistas. Ya hemos mencionado cómo Ryu, el menor, lleva a cabo las responsabilidades domésticas, incluida la vigilancia de su padre para que cumpla con sus obligaciones. De hecho, Ryu afirma que tomó la decisión de irse a vivir con él tras el divorcio de sus padres porque alguien tenía que cuidarle. Mientras que Koichi, su hermano mayor, presenta un rol decididamente activo al autoimponerse de manera obsesiva la misión de reunir a la familia. Se evidencia que los dos asumen roles responsables más propios de una edad adulta que de la despreocupación propia de la infancia, en ambos casos fruto de haber tenido que lidiar prematuramente con problemas del mundo de los adultos.

En esto último hay una sutil diferencia con la tradición del homu dorama. Sin pretender respaldar en su conjunto el retrato antropológico y cultural que Benedict (2010) propusiera del Japón en los años cuarenta -la validez del estudio ha sido ya muy cuestionada-, sí es oportuno indicar cómo el cine coetáneo refleja una de sus observaciones. A saber: que los japoneses tenían una peculiar concepción de la educación infantil, según la cual el niño era dejado en un "estado de excepción” respecto al rígido sistema de normas procedimentales y códigos jerárquicos que caracterizaba a la sociedad de la época. La infancia se desarrollaba en este feliz aislamiento, algo asilvestrado, 
hasta que tenía lugar una entrada en el mundo adulto que sucedía por choque, sin rituales de iniciación previos. Si se observan algunas películas centradas en la temática infantil de Yasujiro Ozu (Nací, pero... [Umarete wa mita keredo, 1932], Buenos días [Ohayo, 1959]) o Hiroshi Shimizu (Niños en el viento [Kaze no naka no kodomo, 1937], Four Seasons of Children [Kodomo no shiki, 1939]), prácticamente coetáneas a los estudios de Benedict, el tratamiento de la relación entre mundo infantil y mundo adulto surge de la confrontación. Los niños crean su propio universo cerrado y con normas específicas, que chocan (y a veces ponen de manifiesto su sinsentido) con las normas del mundo adulto. El resultado final suele ser la integración de lo infantil en lo adulto tras una trama de crecimiento (Santos, 2005, pp. 216-228).

Por el contrario, lo que sucede de forma muy clara en Milagro es que la relación entre los dos mundos es de intercambio bidireccional, con lo que los límites entre ambos quedan muy diluidos. En Nadie sabe, con la mencionada "eliminación" de la esfera adulta, esos límites directamente no existen; en lugar de la interacción de los dos mundos sucede el intento de imitación infantil de un mundo adulto que apenas se manifiesta. Por su parte, lo que en De tal padre, tal hijo tiene lugar es un proceso inverso de integración: lo adulto en lo infantil. Es relevante, por tanto, que una de las diferencias notorias que las películas de Koreeda establecen respecto al homu dorama clásico es el reflejar hibridaciones entre ambos mundos: da cuenta de su voluntad de indagar en lo que parecen aspectos cambiantes (o al menos, poco explorados previamente) de la sociedad japonesa. Lo que apunta, además de a una herencia del viejo género, a su actualización.

\section{Los afectos infantiles}

$\mathrm{Si}$ las relaciones entre personajes adultos en el cine de Koreeda están marcadas por la fragilidad y los conflictos que tienden a separarlos, la forma en que los niños se relacionan ilustra por el contrario una envidiable capacidad de convergencia y sintonía. Cabe afirmar que se da entre ellos una 
afinidad natural que, como vamos a ver, sirve a un propósito temático recurrente: la consideración de la familia como un espacio de relaciones afectivas de apoyo que trasciende el valor de los lazos sanguíneos.

\subsection{La afinidad de los niños}

De las tres películas objeto de estudio, Milagro es la que desarrolla de forma más vivaz esta afinidad natural que distingue a las relaciones infantiles. Este hecho se advierte en primer lugar en la relación de Koichi y Ryu. Los dos hermanos comparten un lenguaje propio, promesas de ayuda, confidencias y disculpas. Y si se enfadan en algún momento, el relato presenta estos pasajes como retazos narcisistas que Koichi deberá dejar atrás en su proceso de formación y aceptación de la realidad del mundo: es lo que sucede cuando no tolera que Ryu no comparta con él su deseo de reunificación familiar, que se haya adaptado con facilidad al nuevo entorno, o que se presente a la cita de los trenes-bala acompañado de sus nuevos amigos.

La armonía de las relaciones infantiles encuentra una colorida extensión precisamente en el retrato coral de los respectivos grupos de amigos de Koichi y Ryu, lo cual hace de Milagro la película más risueña de las tres referidas. El énfasis que Koreeda pone en la aspiración íntima de Koichi, como antes hizo con Akira y sus hermanos en Nadie sabe, encuentra un eco en esta ocasión en la ilustración de los deseos particulares de todos estos niños que rodean a los dos hermanos. Por ingenuas que sus ambiciones puedan ser - hacer dibujos bonitos sin esforzarse, ser el mejor lanzador de peonzas, casarse con una bibliotecaria, etc.-, hay en el film una voluntad de respeto hacia los niños personificada especialmente en Mugami, la niña que reivindica ante su madre la determinación de perseguir su sueño de ser actriz a pesar de que su talento sea dudoso. Al mismo tiempo, el film reconoce cómo el propio devenir de la vida les obligará a los niños a modificar o rebajar sus expectativas. En este sentido, la muerte del perro de uno de ellos y el deseo incumplido de que resucite contribuye al proceso de maduración del protagonista. 
Además de por esta puesta en común de sus deseos, que visualmente se concreta en una pancarta que levantarán cuando se crucen los dos trenesbala, la afinidad infantil es recalcada por otras vías. Aunque Koichi se moleste con Ryu por haber traído a sus nuevos amigos, enseguida las dos cuadrillas convergen en un grupo homogéneo basta con apreciar la recurrencia visual de sus desplazamientos siempre a la carrera, o la gratitud que expresan al unísono ante la cena que les prepara el hospitalario matrimonio de ancianos

Como se verá en el siguiente subepígrafe, es también fuerte la unidad entre los cuatro hermanastros de Nadie sabe; y en De tal padre, tal hijo, Koreeda expresa en diferentes ocasiones un contraste entre los niños de las dos familias que armonizan felizmente en el ámbito del juego, y las tiranteces que se aprecian en las relaciones de los dos matrimonios, cada uno de ellos con sus prevenciones, miedos e intereses particulares. Particularmente ilustrativa es la secuencia en la que los padres negocian con los responsables del hospital la posibilidad del intercambio de hijos y la compensación económica, momento en el que irrumpen los niños y disparan a los adultos con sus pistolas imaginarias, haciéndoles así pagar -cual delegados de la voz autoral de Koreeda- por el “crimen” que están dispuestos a llevar a cabo contra ellos (F7).
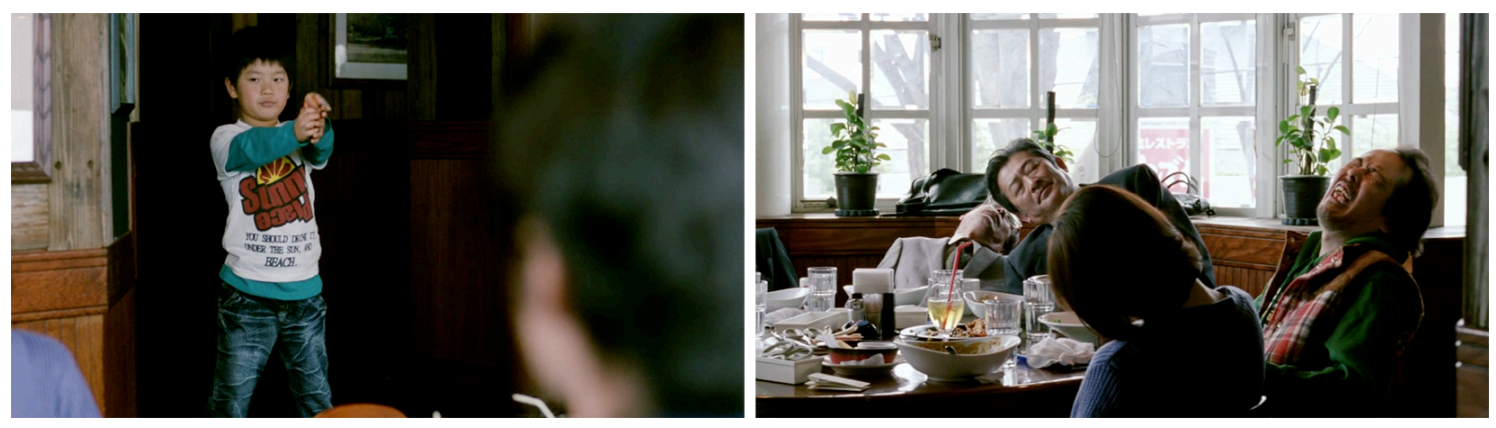

F7. De tal padre, tal hijo (Soshite chichi ni Naru, 2013)

\subsection{Lazos de sangre, lazos de convivencia}

En la filmografía de Koreeda se hace progresivamente evidente la pregunta por qué tipo de vínculos componen los lazos familiares: si son los sanguíneos 
o, en cambio, los que nacen gracias a los afectos que surgen de la convivencia bajo un mismo techo. Existe un posicionamiento explícito por parte del cineasta a favor de la segunda postura, frente a lo común que es en la sociedad japonesa, según él afirma, el énfasis en lo sanguíneo (Levine, 2013). Ya en Maborosi, la mujer protagonista decide no abandonar a su segundo esposo tras comprobar cómo el hijo biológico de ella tiene una relación afectuosa con la hija de él, con la que es, a ojos del niño, su nueva hermana. También Nuestra hermana pequeña incide en la feliz y enriquecedora incorporación de una hermanastra adolescente a un núcleo familiar constituido por tres hermanas naturales y mayores que ella. $\mathrm{Y}$ ya se ha visto cómo De tal padre, tal hijo se edifica en torno a la educación afectiva de un hombre que tiene que aprender a querer genuinamente al niño que ha criado, con independencia de que este no sea de su sangre.

Los cuatro hermanastros de Nadie sabe, como ya hemos detallado, ilustran también la radicalidad de los lazos afectivos que surgen entre los niños con independencia de su ascendencia biológica. Nada tiene más importancia para ellos que preservar la supervivencia de la familia que conforman. Esta decantación se enfatiza además con el personaje de Saki, una adolescente de clase acomodada que, privada del cuidado paterno y víctima de acoso escolar4, encuentra en el hogar precario de Akira y sus hermanastros la compañía y el afecto -la familia- que precisa. El juego (lápiz, papel y tijera) vuelve a ser otro vector de acercamiento entre los niños, cuya solidaridad mutua se subraya con el motivo visual recurrente de darse la mano (Akira a Yuki, Kyoko a Shigeru, Saki a Akira) en situaciones emocionalmente difíciles.

Salvado un contratiempo que la separa de Akira, Saki es bienvenida al espacio doméstico de los cuatro hermanastros, nada celosos de su vínculo. Aunque su relación principal es con Akira, el relato teje un vínculo entre ella y la pequeña Yuki, que anhela -la dibuja, pregunta por ella- la presencia de esta chica, la mayor de todos ellos. Por su parte, Saki le devolverá su afecto

\footnotetext{
4 Aunque, según explicamos, la mirada de Koreeda es mucho más benigna sobre los niños que sobre los adultos no implica esto una ingenuidad por su parte. El acoso escolar que sufre Saki y la crueldad de que es objeto Akira por los amigos que hace, ilustran ciertas patologías sociales propias de los adolescentes.
} 
acariciándole el cabello, habiendo ya Yuki fallecido; y acompañará a Akira a enterrarla. Como evidencia el plano final del film (F8), el vacío que deja la muerte de Yuki es de alguna forma suplantado por la incorporación de Saki al seno familiar. Antes de que el plano se congele, ella y Kyoko conversan con desenfado, vislumbrando una complicidad femenina que años más tarde se recogerá entre las dos madres de De tal padre, tal hijo.

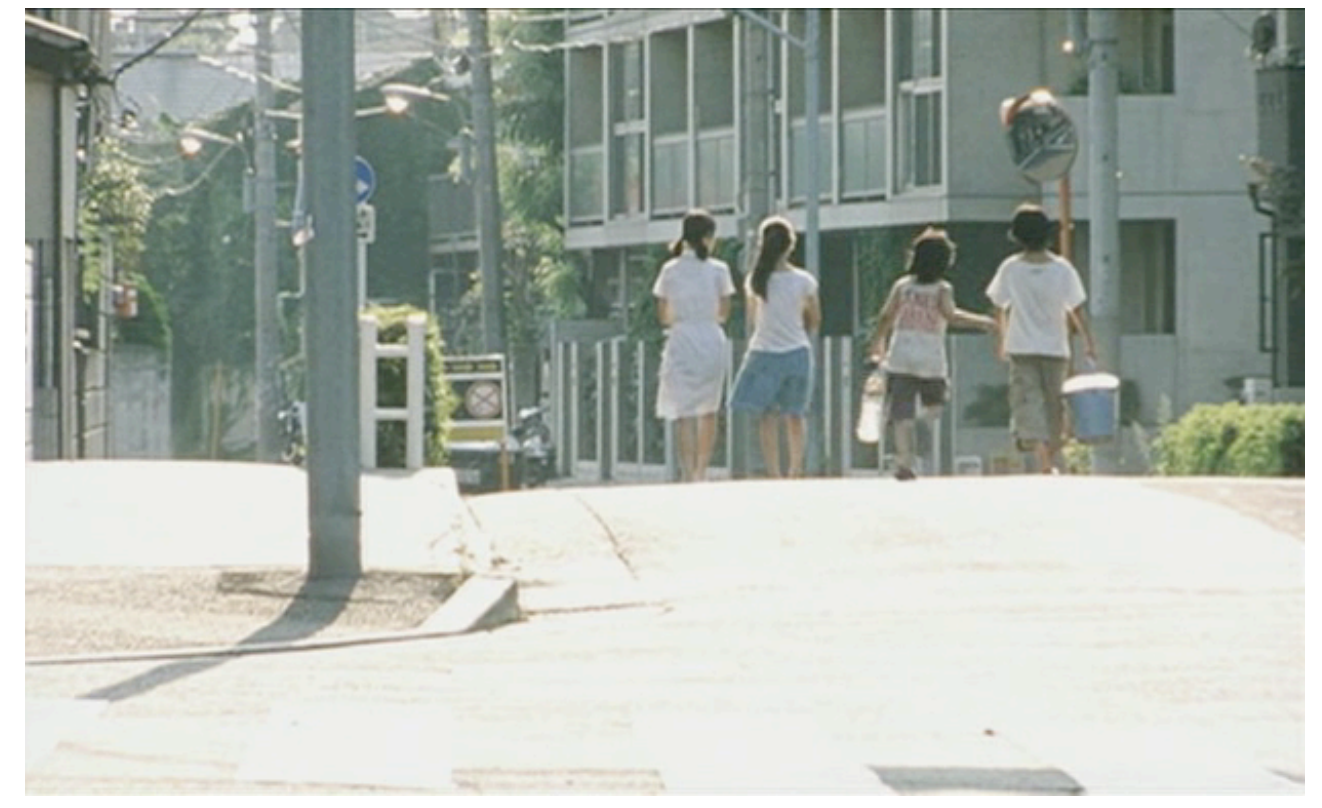

F8. Nadie sabe (Dare mo shiranai, 2004)

En De tal padre, tal hijo, a pesar de que Keita vive con callada consternación la "traición" de su padre, Ryota, sin embargo se atisba como posibilidad su aclimatación al nuevo hogar (que implica también, cabe recordar, un descenso social). Efectivamente, su asimilación podría llegar a ser plena gracias a la atención y a las muestras de cariño que recibe de sus nuevos $-\mathrm{y}$ biológicos- padres, y a la naturalidad con la que se relaciona con sus nuevos hermanos. Esta posibilidad se presenta como un ejemplo más de la fe de Koreeda en la conformación de núcleos familiares sobre la base de los afectos que surgen y se comparten entre sus miembros. 


\section{Conclusión}

De los once largometrajes de ficción que Koreeda ha realizado hasta la fecha, siete se organizan alrededor de la institución familiar y sus conflictos. En Nadie sabe, Milagro y De tal padre, tal hijo, el tiempo plácido de la infancia es desbaratado a raíz de alguna forma de desestructuración familiar de la que son responsables las figuras paternas. Es recurrente en estas películas un contraste entre unos adultos inmaduros que actúan siendo ajenos a las necesidades afectivas de sus hijos, y unos niños que asumen responsabilidades impropias de su edad en el afán de mantener cohesionado el núcleo familiar. Se produce así en estos filmes una hibridación entre las esferas de la infancia y la adultez que colisiona con las tradicionales y rígidas fronteras establecidas entre una y otra en el drama doméstico clásico japonés. Este detalle ejemplifica muy bien cómo las tres películas muestran una notable filiación con aquel homu dorama, pero también, ciertas vías de actualización.

En contraste con la ceguera de los padres en relación con sus hijos, los relatos fílmicos -con la excepción de De tal padre, tal hïo, que alterna la mirada de un padre y un niño- adoptan el punto de vista de los niños. Desde este posicionamiento exploran con delicadeza sus aflicciones y sancionan como legítimo su anhelo de querer recuperar el caudal afectivo sobre el que se sustenta la institución familiar. Surge de este modo una corriente de empatía con unos niños que, a pesar de las circunstancias adversas que padecen, son vistos por Koreeda con luminosidad. Así, con los inevitables matices entre cada película, los niños de Nadie sabe, Milagro y De tal padre, tal hïo son alegres, generosos, agradecidos, cuidan unos de otros y demuestran curiosidad y capacidad de asombro. A ello se suma su gran descomplicación -apoyada con frecuencia en el juego- a la hora de establecer entre ellos relaciones amistosas y fraternas, de lo que se deduce que viven de una manera genuina, a diferencia de los adultos, tantas veces desorientados por motivos sentimentales, mundanos o egoístas. 


\section{Referencias bibliográficas}

Anderson, J. I., Richie, D. (1982). The Japanese film: Art and industry. Princeton: University Press.

Benedict, R. (2010). El crisantemo y la espada: patrones de la cultura japonesa. Madrid: Alianza, 2010.

Bradshaw, P. (2015). "Hirokazu Kore-eda: They compare me to Ozu... But I'm more like Ken Loach". The Guardian. http://www.theguardian.com/film/2015/may/21/hirokazu-koredirector-our-little-sister-interview. Consultado el 4-05-2016.

Caro-Oca, A. M., López-Rodríguez, F. J. (2011). "The Japanese family through Ozu's and Koreeda's cinema”. Global Cinema (Dossier The Ties that Bind). http://www.globalcinema.eu//single.php?sl=FamilyJapan-cinema-Ozu-Koreeda. Consultado el 4-05-2016.

Desser, D. (2007). The Imagination of the Trascendent: Kore-eda Hirokazu's Maborosi (1995). En A. Phillips y J. Stringer (eds.), Japanese cinema: texts and contexts (pp. 273-283). New York: Routledge.

Fecé, J. L. (2003). Paisajes del vacío. Hirokazu Kore-eda. En R. Lardín y J. Sánchez-Navarro (eds.), El principio del fin. Tendencias y efectivos del novísimo cine japonés (pp. 175-195). Barcelona: Paidós Ibérica.

Jacoby, A. (2011). Why nobody knows: family and society in modern Japan. Film Criticism, 35 (2-3), 66-83.

Joo, J.-S., Park, J.-H. (2013). A Study on Hirokazu Kore-eda - The Deepest and Continuous Concerns About the Contemporary Japanese Society. Film Studies, 55, 543-569.

Levine, S. (2013). Interview with Hirokazu Kore-eda, Director of 'Like Father, Like Son'. Indiewire. 21-11-2013. http://blogs.indiewire.com/sydneylevine/interview-with-hirokazukore-eda-director-of-like-father-like-son-international-film-business. Consultado el 4-05-2016.

López Rodríguez, F. J. (2013). La familia japonesa y su representación en el cine de Hirokazu Koreeda. Kokoro: Revista Para La Difusión de La Cultura Japonesa. No extra 1 (Japón: identidad, identidades).

McDonald, K. I. (2006). Reading a Japanese film: Cinema in Context. Honolulu: University of Hawaii Press.

Richie, D. (2005). Cien años de cine japonés. Madrid: Jaguar.

Santos, A. (2005). Yasujiro Ozu: elogio del silencio. Madrid: Cátedra.

Sato, T. (1987). Currents in Japanese cinema: essays. Tokyo, New York: Kodansha International.

Schilling, M. (2011). Kore-eda Hirokazu Interview. Film Criticism Vol. 35, 23 (Special Double Issue: Koreeda Hirokazu), 11-20. 


\section{Películas citadas}

Koreeda, H. (1995). Maboroshi no hikari (Maborosi). Japón: TV Man Union

Koreeda, H. (2004). Dare mo shiranai (Nadie sabe). Japón: Cinequanon, Bandai Visual

Koreeda, H. (2008). Aruitemo, aruitemo (Still Walking). Japón: Cinequanon

Koreeda, H. (2011). Kiseki (Milagro). Japón: Gaga, TV Man Union

Koreeda, H. (2013). Soshite chichi ni naru (De tal padre, tal hijo). Japón: Gaga, TV Man Union

Koreeda, H. (2015). Umimachi Diary (Nuestra hermana pequeña). Japón: Gaga, TV Man Union, Toho

Koreeda, H. (2016). Umi yori mo mada fukaku (Después de la tormenta). Japón: Aoi Promotion, Bandai Visual Company, Fuji Television Network, Gaga

Ozu, Y. (1932). Umarete wa mita keredo (Nací, pero...). Japón: Shochiku

Ozu, Y. (1959). Ohayo (Buenos días). Japón: Shochiku

Shimizu, H. (1937). Kaze no naka no kodomo (Niños en el viento). Japón: Shochiku

Shimizu, H. (1939). Kodomo no shiki (Four Seasons of Children). Japón: Shochiku.

Cómo citar: Echart, P. y Muñoz-Garnica, M. (2017). Infancia y desestructuración familiar en el cine de Hirokazu Koreeda: Nadie sabe, Milagro y De tal padre, tal hijo. Fotocinema. Revista científica de cine $y$ fotografía, $\quad \mathrm{n}^{\mathrm{o}} \quad 14, \quad \mathrm{pp}$. 313-339. Disponible: http://www.revistafotocinema.com/ 